\title{
Multivariate prognostic factors analysis for second-line chemotherapy in advanced biliary tract cancer
}

L Fornaro $^{*}, 1$, S Cereda ${ }^{2}$, G Aprile ${ }^{3}$, S Di Girolamo ${ }^{4}$, D Santini ${ }^{5}$, N Silvestris ${ }^{6}$, S Lonardi ${ }^{7}$, F Leone ${ }^{8}$, M Milella $^{9}$, C Vivaldi ${ }^{10}$, C Belli ${ }^{2}$, F Bergamo ${ }^{7}$, S E Lutrino ${ }^{3}$, R Filippi ${ }^{8}, M_{\text {Russano }}{ }^{5}$, V Vaccaro ${ }^{9}$, A E Brunetti ${ }^{6}$, V Rotella ${ }^{1}$, A Falcone ${ }^{10}$, M A Barbera ${ }^{4}$, J Corbelli ${ }^{4}$, G Fasola ${ }^{3}$, M Aglietta ${ }^{8}$, V Zagonel ${ }^{7}$, M Reni ${ }^{2}$, E Vasile ${ }^{10}$ and G Brandi ${ }^{4}$

${ }^{1}$ Department of Oncology, Unit of Medical Oncology, Azienda USL2 Lucca Via dell'Ospedale 1, 55100 Lucca, Italy; ${ }^{2}$ Department of Medical Oncology, San Raffaele Scientific Institute, 20133 Milano, Italy; ${ }^{3}$ Department of Oncology, University and General Hospital, 33100 Udine, Italy; ${ }^{4}$ Unit of Medical Oncology, Sant'Orsola Malpighi Hospital, University of Bologna, 40138 Bologna, Italy; ${ }^{5}$ Medical Oncology, University Campus Bio-Medico, 00128 Roma, Italy; ${ }^{6}$ Unit of Medical Oncology, National Cancer Institute Giovanni Paolo II, 70124 Bari, Italy; ${ }^{7}$ Unit of Medical Oncology 1, Istituto Oncologico Veneto-IRCCS, 35128 Padova, Italy; ${ }^{8}$ Unit of Medical Oncology, Institute for Cancer Research and Treatment IRCCS Candiolo, University of Torino, 10060 Torino, Italy; ${ }^{9}$ Medical Oncology A, Regina Elena National Cancer Institute, 00144 Roma, Italy and ${ }^{10}$ Unit of Medical Oncology 2, Azienda Ospedaliero-Universitaria Pisana, University of Pisa, 56126 Pisa, Italy

Background: The role of second-line chemotherapy (CT) is not established in advanced biliary tract cancer (aBTC). We investigated the outcome of aBTC patients treated with second-line CT and devised a prognostic model.

Methods: Baseline clinical and laboratory data of 300 consecutive aBTC patients were collected and association with overall survival (OS) was investigated by multivariable Cox models.

Results: The following parameters resulted independently associated with longer OS: Eastern Cooperative Oncology Group performance status of $0(P<0.001$; hazard ratio (HR), 0.348; 95\% confidence interval (Cl) 0.215-0.562), CA19.9 lower than median $(P=0.013 ; \mathrm{HR}, 0.574 ; 95 \% \mathrm{Cl} 0.370-0.891)$, progression-free survival after first-line $\mathrm{CT} \geqslant 6$ months $(P=0.027 ; \mathrm{HR}, 0.633 ; 95 \%$ $\mathrm{Cl} 0.422-0.949)$ and previous surgery on primary tumour ( $P=0.027 ; \mathrm{HR}, 0.609 ; 95 \% \mathrm{Cl} 0.392-0.945)$. We grouped the 249 patients with complete data available into three categories according to the number of fulfilled risk factors: median OS times for good-risk (zero to one factors), intermediate-risk (two factors) and poor-risk (three to four factors) groups were 13.1, 6.6 and 3.7 months, respectively $(P<0.001)$.

Conclusions: Easily available clinical and laboratory factors predict prognosis of aBTC patients undergoing second-line CT. This model allows individual patient-risk stratification and may help in treatment decision and trial design.

Biliary tract cancer (BTC) is an uncommon and heterogeneous tumour family with poor prognosis. Surgery represents the only curative option for these patients in less than $25 \%$ of the cases and the relapse rate is high (de Groen et al, 1999). The majority of these patients have advanced disease (metastatic or unresectable) at diagnosis and median overall survival (OS) rarely exceeds 6-8 months (Charbel and Al-Kawas, 2011). In this scenario, palliative treatment aims at improving quality of life and OS. In particular, in randomised phase III trials systemic chemotherapy (CT) was associated with a significant benefit in terms of quality and length

*Correspondence: Dr L Fornaro; E-mail: lorenzo.fornaro@gmail.com

Received 24 November 2013; revised 9 March 2014; accepted 17 March 2014; published online 8 April 2014

(c) 2014 Cancer Research UK. All rights reserved 0007-0920/14 
of life compared with best supportive care alone (Glimelius et al, 1996; Sharma et al, 2010). Subsequently, two randomised studies identified the cisplatin plus gemcitabine regimen as the standard first-line CT (Okusaka et al, 2010; Valle et al, 2010): in these trials, OS was 11.7 and 11.2 months in the combination arm vs 8.1 and 7.7 months for single agent CT $(P<0.001)$, respectively. The combination of gemcitabine and oxaliplatin is another regimen which resulted active and well tolerated in prospective trials in BTC, and it is thus largely used in clinical practice (André et al, 2004).

Up to $50 \%$ of BTC patients failing standard first-line therapy maintain a good performance status (PS) and remain eligible for further treatment (Cereda et al, 2013). Nevertheless, clinical trials focused on second-line therapy are difficult to perform due to the rarity of these tumours and the heterogeneity of patient population, which is generally characterised by refractoriness to platinum compounds and gemcitabine. So, in the absence of randomised studies, only limited and disappointing data regarding second-line CT regimens and biologic agents are available in the literature with objective response rates (RRs) of $0-23 \%$ and median progression-free survival (PFS) times not exceeding 4 months (Furuse et al, 2012; Lee et al, 2012; Bridgewater et al, 2013; Cereda et al, 2013; Walter et al, 2013). Furthermore, identification of predictive or prognostic factors able to identify patients who could benefit from second-line therapy is a clinical issue (Lee et al, 2012; Cereda et al, 2013; Walter et al, 2013).

Herein, we report the results of the largest survey evaluating the clinical practice of several Italian oncologic centres in the treatment of patients with advanced BTCs (aBTCs) relapsed or progressed after a first-line treatment strategy.

\section{MATERIALS AND METHODS}

Patients and methods. We retrospectively identified patients with aBTCs treated with second-line CT at 10 Italian Institutions between 2004 (when initial data with gemcitabine plus a platinum derivative such as oxaliplatin as first-line CT were published (André et al, 2004)) and 2013. Criteria for case inclusion were: cyto-histologically confirmed diagnosis of non-resectable, recurrent or metastatic biliary tract adenocarcinoma (intrahepatic or extrahepatic cholangiocarcinoma, gallbladder and ampullary carcinoma); age $\geqslant 18$ years; confirmed progression after first-line CT, administered until disease progression, unacceptable toxicity, patient refusal or for a maximum of 6 months of treatment; availability of clinico-pathological and laboratory features at the beginning of second-line CT and subsequent response evaluation and survival data. All the patients fulfilling the abovementioned criteria were included in the analyses, independently of the firstand second-line CT regimens received.

Factors included in the univariate analyses for PFS and OS were the following: age; sex; Eastern Cooperative Oncology Group (ECOG) PS (0 vs 1-2); tumour location (intrahepatic vs extrahepatic $v s$ gallbladder $v s$ ampullary); surgery on primary tumour; disease extent (locally advanced $v s$ metastatic); number of disease sites; presence of liver, lung, peritoneal or bone metastases; first-line CT containing gemcitabine and a platinum derivative; objective response and PFS after first-line CT; carcinoembrionic antigen and carbohydrate antigen 19.9 (CA19.9) levels; haematologic parameters (i.e. haemoglobin, white cell count and platelet count); serum albumin; transaminases (alanine aminotransferase and aspartate aminotransferase); alkaline phosphatase; total bilirubin; weight loss $>5 \%$ before second-line CT. Laboratory variables were initially recorded as continuous variables and later dichotomised according to the median value of each variable. Haematologic parameters were dichotomised using local laboratory values to define anaemia $\left(12 \mathrm{~g} \mathrm{dl}^{-1}\right)$, leukocytosis $\left(10000 \mathrm{~mm}^{-3}\right)$ and thrombocytosis $\left(400000 \mathrm{~mm}^{-3}\right)$. The threshold of 70 years of age was used to identify elderly patients. PFS after first-line CT was measured from the date of treatment beginning to the date of progression; to accurately evaluate the association of first-line PFS with OS after second-line CT, this variable was dichotomised, too, using as cut-off point the median first-line PFS of the entire study population.

All the patients included provided written informed consent before treatment initiation, allowing treating physicians to administer the proposed second-line CT, perform laboratory analyses and collect all the data in a site-specific database. The different data sets from each centres have been later merged and information shared among centres, assuring patient anonymisation.

Statistical analysis. The primary end point of this retrospective analysis was OS. OS was measured from the date of the first cycle of second-line CT to the date of death or last follow-up visit. Secondary end points were: objective tumour response, evaluated by Response Evaluation Criteria in Solid Tumors (RECIST) v. 1.0 (Therasse et al, 2000) (imaging was repeated every 10 weeks until disease progression) and PFS (measured from the date of the first cycle of second-line CT to the date of disease progression or death, whichever occurred first). PFS and OS were estimated using the Kaplan-Meier product-limit method.

For both second-line PFS and OS, we initially performed a univariate assessment of the prognostic effect of each factor, comparing survival curves by the use of the log-rank test. In order to account for multiple testing, a two-sided $P$-value of $<0.005$ was considered significant at univariate analysis. Multivariate analysis was then carried out using stepwise Cox proportional hazards regression modelling, stratifying for the second-line CT regimens received (monotherapy vs combination $\mathrm{CT}$ ) and setting statistical significance at $P<0.05$ for a two-sided test. Statistical analyses were carried out using the statistical software package SPSS 19.0 (SPSS, Chicago, IL, USA).

\section{RESULTS}

Patients. Of the 811 patients starting first-line CT, 357 patients (44\%) were treated with second-line CT from 04 March 2004 to 10 April 2013. Three hundred patients (37\%) fulfilled all the abovementioned criteria and were therefore included in the analysis. Patient characteristics are summarised in Table 1. Fiftytwo percent of patients had an intrahepatic cholangiocarcinoma, $21 \%$ had an extrahepatic cholangiocarcinoma, whereas gallbladder and ampullary carcinoma were recorded in 18 and $9 \%$ of the cases, respectively. More than a half (64\%) of patients received a first-line gemcitabine plus platinum (either oxaliplatin or cisplatin) combination: with regard to first-line therapy, partial response was reported in 55 patients (18\%; RR: 18\%), whereas median PFS was 6.0 months.

Second-line treatment outcome. Second-line CT regimens are listed in Table 1. Among the 293 evaluable patients, 12 (4\%) partial responses (RR: $4 \%)$ and $88(30 \%)$ disease stabilisations have been observed, for a disease control rate (DCR) of $34 \%$. At the time of the analysis, 270 patients (90\%) had progressed and median PFS was 3.2 months (95\% confidence interval (CI): 2.9-3.5).

We exploratory investigated factors associated with better outcome in terms of objective response and PFS with second-line CT. In particular, we queued whether different second-line CT regimens achieved different outcome, and found that the use of combination regimens seemed associated with higher DCR compared with monotherapy (39\% vs $26 \% ; P=0.030)$ as well as longer median PFS (3.4 vs 3.0 months; hazard ratio (HR), 0.688; 
Table 1. Patient characteristics $(N=300)$

\begin{tabular}{|c|c|}
\hline & $N(\%)$ \\
\hline Age, years (median, range) & $64(28-85)$ \\
\hline \multicolumn{2}{|l|}{ Gender } \\
\hline $\begin{array}{l}\text { Female } \\
\text { Male }\end{array}$ & $\begin{array}{l}136(45 \%) \\
164(55 \%)\end{array}$ \\
\hline
\end{tabular}

\section{ECOG performance status}

\begin{tabular}{|r|r|}
\hline 0 & $175(58 \%)$ \\
1 & $96(32 \%)$ \\
2 & $29(10 \%)$
\end{tabular}

\section{Primary tumour site}

\begin{tabular}{|l|r|}
\hline Intrahepatic & $157(52 \%)$ \\
Extrahepatic & $64(21 \%)$ \\
Gallbladder & $53(18 \%)$ \\
Ampullary & $26(9 \%)$
\end{tabular}

\section{Surgery for primary tumour}

\begin{tabular}{|l|l|}
\hline Yes & $167(56 \%)$ \\
\hline
\end{tabular}

No

$133(44 \%)$

\section{Previous adjuvant $\mathrm{CT}$}

\begin{tabular}{|l|c|}
\hline Yes & $61(20 \%)$ \\
No & $230(77 \%)$ \\
Not reported & $9(3 \%)$ \\
\hline
\end{tabular}

\section{Previous adjuvant radiotherapy}

\begin{tabular}{l|c} 
Yes & $20(7 \%)$ \\
No & $267(89 \%)$ \\
Not reported & $13(4 \%)$
\end{tabular}

\section{Number of metastatic sites}

\begin{tabular}{|l|r|}
\hline $\begin{array}{l}\text { Median } \\
\text { Range }\end{array}$ & \multicolumn{1}{|c|}{$1-5$} \\
\hline Liver metastases & $250(83 \%)$ \\
\hline $\begin{array}{l}\text { Yes } \\
\text { No }\end{array}$ & $50(17 \%)$ \\
\hline Lung metastases & $92(31 \%)$ \\
\hline Yes & $208(69 \%)$ \\
\hline No &
\end{tabular}

\section{Peritoneal metastases}

\begin{tabular}{|l|r}
\hline Yes & $95(32 \%)$ \\
\hline
\end{tabular}

No

$205(68 \%)$

\section{Bone metastases}

\begin{tabular}{|l|r}
\hline Yes & $31(10 \%)$ \\
\hline
\end{tabular}

No

$269(90 \%)$

\section{Extent of disease}

Locally advanced

Metastatic

$38(13 \%)$

$262(87 \%)$

\section{First-line $\mathrm{CT}$ regimen}

Gemcitabine + platinum

Gemcitabine + fluoropyrimidine

Other gemcitabine-containing regimens

Gemcitabine monotherapy

Other

\section{Table 1. (Continued)}

\begin{tabular}{|c|c|}
\hline & $N(\%)$ \\
\hline \multicolumn{2}{|l|}{ Best objective response with first-line CT } \\
\hline Complete response & $5(1 \%)$ \\
\hline Partial response & 50 (17\%) \\
\hline Stable disease & $123(41 \%)$ \\
\hline Progressive disease & $113(38 \%)$ \\
\hline Not reported & $9(3 \%)$ \\
\hline Median PFS with first-line CT (months) & 6.0 \\
\hline \multicolumn{2}{|l|}{ Second-line $\mathrm{CT}$ regimen } \\
\hline Platinum-based & $96(32 \%)$ \\
\hline Gemcitabine + fluoropyrimidine & $44(15 \%)$ \\
\hline Gemcitabine monotherapy & $22(7 \%)$ \\
\hline Fluoropyrimidine monotherapy & $74(25 \%)$ \\
\hline Irinotecan-containing regimens & $22(7 \%)$ \\
\hline Taxane-containing regimens & $11(4 \%)$ \\
\hline Other & $31(10 \%)$ \\
\hline \multicolumn{2}{|l|}{ Complete blood count } \\
\hline WBC count $>10000 \mathrm{~mm}^{-3}$ & $51(17 \%)$ \\
\hline Haemoglobin $<12 \mathrm{~g} \mathrm{dl}^{-1}$ & $133(44 \%)$ \\
\hline Platelet count $>400000 \mathrm{~mm}^{-3}$ & 20 (7\%) \\
\hline \multicolumn{2}{|l|}{ Blood chemistry } \\
\hline Alkaline phosphatase, $\mathrm{UI}^{-1}$ (median, range) & $247(36-1676)$ \\
\hline $\mathrm{ALT}, \mathrm{UI}^{-1}$ (median, range) & $34(5-503)$ \\
\hline AST, $\mathrm{UI}^{-1}$ (median, range) & $35(12-279)$ \\
\hline Total bilirubin, $\mathrm{mg} \mathrm{dl}^{-1}$ (median, range) & $0.66(0.20-8.00)$ \\
\hline Albumin, $\mathrm{g} \mathrm{dl}^{-1}$ (median, range) & $3.56(2.00-4.58)$ \\
\hline Carcinoembrionic antigen, $\mathrm{ng} \mathrm{ml}^{-1}$ (median, range) & $5(0-1497)$ \\
\hline CA19.9, $\mathrm{U} \mathrm{ml}^{-1}$ (median, range) & $152(0.5-104136)$ \\
\hline
\end{tabular}

95\% CI 0.504-0.850; $P=0.002)$ and OS (8.2 vs 5.2 months; HR, 0.718; 95\% CI 0.535-0.964; $P=0.028)$. At univariate analysis, factors associated with longer PFS were: ECOG PS 0 $(P<0.001)$, locally advanced disease $(P=0.001)$, first-line PFS $\geqslant 6$ months $(P=0.004)$, CA19.9 $\leqslant 152 \mathrm{U} \mathrm{ml}^{-1}(P<0.001)$ and WBC count $\leqslant 10000 \mathrm{~mm}^{-3}(P<0.001)$ (Supplementary Table 1$)$. When tested at multivariate analysis, stratifying for the second-line CT received, the following parameters retained statistical significance as good prognostic factors: WBC count $\leqslant 10000 \mathrm{~mm}^{-3}$ $(P=0.029 ; \quad H R, 0.659 ; 95 \%$ CI 0.454-0.957), ECOG PS 0 $(P=0.030 ; \mathrm{HR}, 0.701 ; 95 \%$ CI $0.509-0.965), \mathrm{CA} 19.9 \leqslant 152 \mathrm{U} \mathrm{ml}^{-1}$ $(P=0.028$; HR, 0.726; 95\% CI 0.545-0.966) and first-line PFS $\geqslant 6$ months $(P=0.040$; HR, 0.748; 95\% CI 0.567-0.987).

Multivariate prognostic model. At the time of the analysis, 224 patients (75\%) have died and median OS from the beginning of second-line CT for the entire population was 7.2 months (95\% CI: 6.0-8.4) (Figure 1A).

At univariate analysis (Supplementary Table 2), 10 variables were significantly associated with longer OS: ECOG PS 0 $(P<0.001)$, site of primary tumour $(P=0.003)$, surgery on primary tumour $(P<0.001)$, first-line PFS $\geqslant 6$ months $(P=0.003)$, CA19.9 $\leqslant 152 \mathrm{U} \mathrm{ml}^{-1}(P<0.001)$, haemoglobin $\leqslant 12 \mathrm{~g} \mathrm{dl}^{-1}(P=0.001)$, WBC count $\leqslant 10000 \mathrm{~mm}^{-3} \quad(P<0.001)$, serum albumin $\leqslant 3.56 \mathrm{~g} \mathrm{dl}^{-1} \quad(P<0.001)$, alkaline phosphatase $\leqslant 247 \mathrm{Ul}^{-1}$ $(P=0.004)$ and weight loss $>5 \%(P<0.001)$.

Multivariate regression analysis (Table 2) was therefore conducted including all the abovementioned parameters, 
A
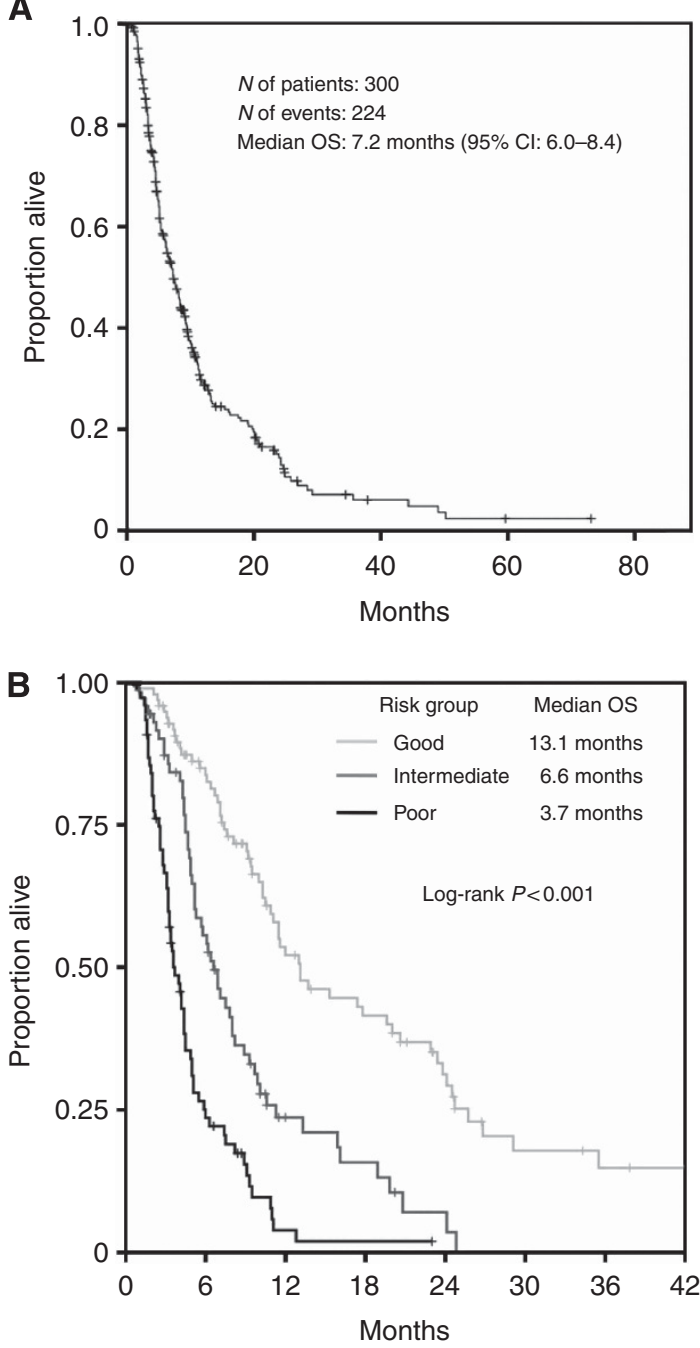

Figure 1. Survival curves for all patients (A) and according to the risk groups identified at multivariate analysis (B). Abbreviations: $N=$ number; $O S=$ overall survival.

\section{Table 2. Multivariate analysis}

\begin{tabular}{|l|c|c|c|}
\hline & HR & $\mathbf{9 5 \%} \mathbf{C l}$ & $\boldsymbol{P}$-value \\
\hline PFS after first-line CT $\geqslant 6$ months & 0.633 & $0.422-0.949$ & 0.027 \\
\hline Previous surgery on primary tumour & 0.609 & $0.392-0.945$ & 0.027 \\
\hline Pretreatment CA19.9 $\leqslant 152 \mathrm{U} \mathrm{ml}^{-1}$ & 0.574 & $0.370-0.891$ & 0.013 \\
\hline ECOG performance status 0 & 0.348 & $0.215-0.562$ & $<0.001$ \\
\hline
\end{tabular}

Abbreviations: $\mathrm{Cl}=$ confidence interval; $\mathrm{CT}=$ chemotherapy; $\mathrm{ECOG}=$ Eastern Cooperative Oncology Group; $\mathrm{HR}=$ hazard ratio; PFS = progression-free survival.

stratifying for the second-line CT received. Independent, favourable prognostic factors were: an ECOG PS of $0(P<0.001$; HR, 0.348 ; $95 \%$ CI $0.215-0.562)$, CA19.9 $\leqslant 152 \mathrm{U} \mathrm{ml}^{-1}(P=0.013$; HR, $0.574 ; 95 \%$ CI $0.370-0.891$ ), previous surgery on primary tumour $(P=0.027$; HR, 0.609; 95\% CI 0.392-0.945) and PFS after first-line $\mathrm{CT} \geqslant 6$ months $(P=0.027$; HR, 0.633; 95\% CI 0.422-0.949).

We depicted a multivariate prognostic model by combining these four independent prognostic factors according to the following criteria: good-risk group, that is, patients with zero to one negative prognostic factors; intermediate-risk group, that is, patients with two negative prognostic factors; poor-risk group, that is, patients with three to four negative prognostic factors. Of 249 patients with complete data for the four variables, 98 patients were categorised as good-risk group, 73 patients as intermediate-risk group and 78 patients as poor-risk group. The survival curves according to the prognostic model are provided in Figure 1B. Median OS for good-, intermediate- and poor-risk groups were 13.1 months (95\% CI 9.0-17.2), 6.6 months (95\% CI 5.2-8.0) and 3.7 months (95\% CI 3.0-4.4), respectively. Survival differences among groups achieved statistical significance $(P<0.001)$.

\section{DISCUSSION}

Second-line treatment of aBTC represents a great challenge: consistent evidence of a survival benefit for palliative CT is lacking, and thus no standard regimen is available in clinical practice (Cereda et al, 2013). The current analysis identified several reliable prognostic factors in this setting. Of note, as it is based on easily available variables, this model has the potentials for widespread use.

To our knowledge, we collected the so far largest series of aBTC patients treated with second-line CT since 2004, when the results of the GEMOX regimen were published (André et al, 2004). Our population is therefore enriched with patients treated with a platinum plus gemcitabine combination in first-line, which is considered the standard approach after the publication of the ABC-02 and BT trials (Okusaka et al, 2010; Valle et al, 2010). Results of first-line CT are in line with literature data both in terms of DCR and PFS, thus reassuring about the risk of major selection bias in case collection. Our series confirms that almost half of aBTC cases received second-line therapy and that CT has only marginal activity (RR: 4\%) in pretreated patients, even though it may offer disease control in up to $34 \%$ of the cases. By the identification of four clinical and laboratory parameters such as ECOG PS, basal CA19.9 level, previous surgery on primary tumour and first-line PFS, three patient subgroups with different survival outcome can be identified. The OS curves for these risk groups are comparable with those reported by Park et al (2009) in the firstline setting, suggesting that the magnitude of benefit of salvage treatment is predictable, too.

PS confirmed to be the most important prognostic determinant even in pretreated aBTC patients. This observation is in line with other reports in the first- and second-line setting (Park et al, 2009; Bridgewater et al, 2013; Cereda et al, 2013). As aBTC is a rapidly progressing disease, which may impact on patients' general condition and liver function, an accurate evaluation of pretreatment PS is essential before any therapeutic decision.

The prognostic value of CA19.9 in our series is apparently in contrast with the results reported in first-line (Park et al, 2009). As the expression of tumour markers widely varies among patients, this discrepancy may be partly explained by different patient populations. CA19.9 is a proven prognostic factor in other gastrointestinal malignancies such as gastric and pancreatic cancer, and our data are in line with these experiences. As we did not find a significant impact of the number of metastatic sites on OS, higher CA19.9 levels may not only reflect a higher tumour burden, but also a more aggressive tumour behaviour.

The positive prognostic role of previous surgery on primary tumour is probably linked to a more favourable disease course in patients presenting with resectable tumour; in fact, biology may differ in localised and unresectable or metastatic disease at diagnosis. Moreover, immediate surgery may avoid otherwise frequent complications in aBTC such as biliary or bowel obstruction, thus favourably impacting on patient conditions and treatment tolerability.

Walter et al (2013) reported in a smaller series that PFS after firstline CT is a predictor of benefit from second-line therapy in terms of 
PFS; however, they did not analyse OS or integrate this parameter into a prognostic score. Similarly, Lee et al (2012) found that response to first-line $\mathrm{CT}$ is an independent predictor of OS with salvage therapy in their series of 89 cases. Even though the formal demonstration that PFS is a surrogate for OS in aBTC is lacking, it is arguable that PFS may represent an index of the chemosensitivity of the disease. Therefore, no or limited benefit from first-line CT should discourage from an aggressive approach in second-line. The threshold of 6 months for the PFS variable appears a reasonable choice, as reported median PFS times with first-line platinum plus gemcitabine regimens are in the range 5.7-8.0 months (Okusaka et al, 2010; Valle et al, 2010; Furuse et al, 2012).

Our retrospective study did not aim to identify an optimal second-line CT regimen. Univariate analysis suggests that combination CT is associated with a slightly higher DCR compared with monotherapy ( $39 \%$ vs $26 \% ; P=0.002)$ as well as slightly longer median PFS (3.4 vs 3.0 months; $P=0.030$ ), as reported also by Walter et al (2013), and OS (8.2 vs 5.2 months; $P=0.028)$. We thus selected the second-line CT regimen as a stratification factor at multivariate analysis, in order to develop a prognostic model based only on baseline treatment-independent, patient- and disease-related factors. Therefore, caution is needed and randomised trials are ongoing in order to establish the optimal secondline regimen in aBTC (Cereda et al, 2013). In our opinion, until the results of these trials are made available, the use of combination CT is justified in those patients with good PS and preserved liver function who derived significant benefit from first-line CT (e.g., objective response and PFS longer than 6 months) without major toxicities. This simple prognostic model may thus help in designing and interpreting the results of future trials.

Our analysis confirms that it is unlikely that CT alone could have a dramatic impact on aBTC prognosis. There is, therefore, an urgent need for the identification of molecular targets and effective targeted agents in this setting. Little is known about the molecular basis of aBTC, a wide-spectrum category, which comprises different entities such as cholangiocarcinoma, gallbladder and ampullary cancer. Up to now, several phase II studies evaluated different agents with conflicting results (Cereda et al, 2013; Marino et $a l, 2013)$. In the absence of any treatment individualisation strategy by means of biologic predictive factors, the road toward any improvement in patient outcome appears still too long and windy, and notwithstanding even more necessary. With this regard, it should be kept in mind that the current analysis is based on patients treated with different CT regimens only; therefore, conclusions may not be valid in patients treated with biologics.

In conclusion, our model is based on some easily available clinical and laboratory factors, which reliably identify different groups of aBTC patients with different outcome after second-line CT. If validated in other data sets, these results may represent a useful tool for individual patient-risk evaluation and trial design and interpretation. Results of phase III randomised trials comparing different second-line single agent and combination CT after the failure of a platinum plus gemcitabine regimen are eagerly awaited. Finally, further research on the biologic landscape of BTC and the development of targeted agents are highly warranted in order to move forward the therapeutic armamentarium against this aggressive disease.

\section{CONFLICT OF INTEREST}

The authors declare no conflict of interest.

\section{REFERENCES}

André T, Tournigand C, Rosmorduc O, Provent S, Maindrault-Goebel F, Avenin D, Selle F, Paye F, Hannoun L, Houry S, Gayet B, Lotz JP, de Gramont A, Louvet C. GERCOR Group (2004) Gemcitabine combined with oxaliplatin (GEMOX) in advanced biliary tract adenocarcinoma: a GERCOR study. Ann Oncol 15(9): 1339-1343.

Bridgewater J, Palmer D, Cunningham D, Iveson T, Gillmore R, Waters J, Harrison M, Wasan H, Corrie P, Valle J (2013) Outcome of second-line chemotherapy for biliary tract cancer. Eur J Cancer 49(6): 1511.

Cereda S, Belli C, Rognone A, Mazza E, Reni M (2013) Second-line therapy in advanced biliary tract cancer: what should be the standard? Crit Rev Oncol Hematol 88(2): 368-374.

Charbel H, Al-Kawas FH (2011) Cholangiocarcinoma: epidemiology, risk factors, pathogenesis, and diagnosis. Curr Gastroenterol Rep 13(2): 182-187.

de Groen PC, Gores GJ, LaRusso NF, Gunderson LL, Nagorney DM (1999) Biliary tract cancers. N Engl J Med 341(18): 1368-1378.

Furuse J, Kasuga A, Takasu A, Kitamura H, Nagashima F (2012) Role of chemotherapy in treatments for biliary tract cancer. J Hepatobiliary Pancreatic Sci 19(4): 337-341.

Glimelius B, Hoffman K, Sjödén PO, Jacobsson G, Sellström H, Enander LK, Linné T, Svensson C (1996) Chemotherapy improves survival and quality of life in advanced pancreatic and biliary cancer. Ann Oncol 7(6): 593-600.

Lee SC, Kim K, Kim H, Kim HJ, Kim SH, Bae SB, Kim CK, Lee N, Lee KT, Park SK, Won JH, Yun J, Hong DS, Park HS (2012) Prognostic factor analysis of second-line chemotherapy in advanced biliary tract cancer. $J$ Clin Oncol 30: abstr e14688.

Marino D, Leone F, Cavalloni G, Cagnazzo C, Aglietta M (2013) Biliary tract carcinomas: from chemotherapy to targeted therapy. Crit Rev Oncol Hematol 85(2): 136-148.

Okusaka T, Nakachi K, Fukutomi A, Mizuno N, Ohkawa S, Funakoshi A, Nagino M, Kondo S, Nagaoka S, Funai J, Koshiji M, Nambu Y, Furuse J, Miyazaki M, Nimura Y (2010) Gemcitabine alone or in combination with cisplatin in patients with biliary tract cancer: a comparative multicenter study in Japan. Br J Cancer 103(4): 469-474.

Park I, Lee JL, Ryu MH, Kim TW, Sook Lee S, Hyun Park D, Soo Lee S, Wan Seo D, Koo Lee S, Kim MH (2009) Prognostic factors and predictive model in patients with advanced biliary tract adenocarcinoma receiving first-line palliative chemotherapy. Cancer 115(18): $4148-4155$.

Sharma A, Dwary AD, Mohanti BK, Deo SV, Pal S, Sreenivas V, Raina V, Shukla NK, Thulkar S, Garg P, Chaudhary SP (2010) Best supportive care compared with chemotherapy for unresectable gall bladder cancer: a randomized controlled study. J Clin Oncol 28(30): 4581-4586.

Therasse P, Arbuck SG, Eisenhauer EA, Wanders J, Kaplan RS, Rubinstein L, Verweij J, Van Glabbeke M, van Oosterom AT, Christian MC, Gwyther SG (2000) New guidelines to evaluate the response to treatement in solid tumors. EORTC, NCI USA, NCI Canada. J Natl Cancer Inst 92(3): 205-216.

Valle J, Wasan H, Palmer DH, Cunningham D, Anthoney A, Maraveyas A, Madhusudan S, Iveson T, Hughes S, Pereira SP, Roughton M, Bridgewater J. ABC-02 Trial Investigators (2010) Cisplatin plus gemcitabine versus gemcitabine for biliary tract cancer. $N$ Engl J Med 362(14): 1273-1281.

Walter T, Horgan AM, McNamara M, McKeever L, Min T, Hedley D, Serra S, Krzyzanowska MK, Chen E, Mackay H, Feld R, Moore M, Knox JJ (2013) Feasibility and benefits of second-line chemotherapy in advanced biliary tract cancer: a large retrospective study. Eur J Cancer 49(2): 329-335.

This work is published under the standard license to publish agreement. After 12 months the work will become freely available and the license terms will switch to a Creative Commons AttributionNonCommercial-Share Alike 3.0 Unported License. 